\title{
Post-traumatic stress reactions in survivors of the 2011 massacre on Utøya Island, Norway ${ }^{\dagger}$
}

\author{
Grete Dyb, Tine K. Jensen, Egil Nygaard, Øivind Ekeberg, Trond H. Diseth, Tore Wentzel-Larsen
} and Siri Thoresen

\section{Background}

Although youths in many countries have been exposed to terrorism, few studies have examined early risk and protective factors for the subsequent development of mental health problems.

\section{Aims}

To investigate the levels of post-traumatic stress in survivors of the 2011 massacre on Utøya Island compared with the general population in Norway, and to identify predictive factors.

\section{Method}

Four hundred and ninety survivors were invited to participate. Structured face-to-face interviews were performed 4-5 months after the attack.

\section{Results}

There were 325 study participants (response rate 66\%).
Survivors had been highly exposed to danger and loss. Post-traumatic stress levels were more than six times higher in survivors than in the general population. Predictors were female gender, minority ethnic status, high level of trauma exposure, pain, the loss of someone close and social support.

\section{Conclusions}

Survivor characteristics that can be assessed in the early aftermath of a terrorist attack strongly predict the subsequent mental health problems of exposed youths. The highly elevated symptoms observed were largely attributable to the traumatic experience and reflect the mental health costs of the terrorist attack.

\section{Declaration of interest}

None.
Terrorist attacks are traumatic events that violate security and feelings of safety. They have had an enormous impact throughout the world in terms of loss of life and damage. ${ }^{1}$ Research performed after terror attacks suggests that the prevalence of post-traumatic stress reactions among people with high levels of exposure was substantial and was associated with a wide range of factors such as sociodemographic and background factors, event-exposure characteristics, the loss of life of significant others, physical injuries and social support. ${ }^{2-5}$ After the Beslan school terrorist attack in North Ossetia in Russia in 2004, 50\% of the children who were inside the school during the attack met criteria for post-traumatic stress disorder (PTSD) 3 years later. Exposure and gender predicted the level of distress. ${ }^{6}$ Similarly, studies performed after school shootings show that approximately a third of exposed youths developed PTSD. ${ }^{7-10}$ After the 2007 school shooting in Finland, high levels of post-traumatic distress were reported by $27 \%$ of the females and $7 \%$ of the males, 4 months after the shooting. In line with former studies, levels of exposure to danger and female gender increased the risk of developing post-traumatic stress reactions. ${ }^{11}$ The literature is more inconclusive regarding age as a risk factor for PTSD. ${ }^{12,13}$ In early studies of shootings, peritraumatic emotional reactions were shown to be even more predictive than were the levels of objective trauma exposure. ${ }^{7}$ Unfortunately, this line of research has not been pursued to the same degree as other predictors of post-traumatic stress. ${ }^{14}$ Perceived social support is consistently associated with mental health, and within traumatic stress research, social support has proved to be a protective factor against the development of mental health problems subsequent to trauma. ${ }^{10,15-17}$ However, we do not know the extent of the effect of social support. It may be that social support has a limited buffering effect when the event involves gross atrocities.

†See editorial, pp. 329-330, this issue.
Sadly, although many countries and communities are exposed to terrorist attacks, few studies have been able to identify early predictors of the risk of development of mental health problems after such incidents. ${ }^{18}$ A better understanding of the development of post-traumatic stress reactions is important and may aid professionals in determining who would be in need of early intervention efforts in the community.

\section{The terrorist attack}

On 22 July 2011, Norway experienced two terrorist attacks: first, a bomb exploded in central Oslo, outside the main government building; and second, there was a shooting on Utøya Island, which is located outside Oslo and is a summer camp for the Norwegian Labor Party's youth organisation. At the time of the shooting, 564 people were on the island; the terrorist killed 69, and 56 were admitted to hospital for severe injuries. Several additional factors amplified the atrocity of this attack. First, the youths were isolated on this small island for over $1 \mathrm{~h}$ and $20 \mathrm{~min}$ as the terrorist hunted down and shot them; they had no possibility of escape other than swimming to the mainland across the cold fjord, with the risk of drowning. Second, the terrorist was extremely brutal, often shooting the victims several times, thus leaving many youths having witnessed extreme trauma. Many of the youths knew one another, and they were affiliated through their youth organisation; thus, they lost friends and acquaintances. Moreover, the terrorist used his disguise as a policeman to lure the youths out of their hiding places. This left many youths in prolonged fear, as they did not know whom to trust when the first rescuers came to their aid. During the next few days, survivors returned to their home communities throughout Norway and a national intervention strategy for affected families was implemented in healthcare services.

Two characteristics of the Utøya Island massacre make it suitable for the study of early risk and protective factors: the 
exposure level was presumably approximately the same for all participants, as they were all exposed to a life-threatening situation; further, all survivors could be identified. Most studies of disasters and terrorist attacks face sampling problems, and include participants whose levels of traumatic exposure vary greatly. By contrast, the exposure level was relatively uniform in this group, allowing us to examine the relative importance of other factors for long-term mental health.

The aim of this study was twofold:

(a) to investigate the levels of post-traumatic stress reactions in survivors of the 22 July terrorist attack on Utøya Island in Norway, and compare them with the levels of reactions to the terror attacks in the general population in Norway;

(b) to assess potential predictors of post-traumatic stress reactions among the survivors $4-5$ months after the terrorist attack, with a focus on event characteristics, peritraumatic reactions, age, gender and perceived social support.

\section{Method}

\section{Participants and procedures}

The police registered 495 survivors of the terrorist attack on Utøya Island. Three months after the terrorist attack, 490 survivors who were at least 13 years of age were sent postal invitations to participate, and were subsequently contacted by telephone. Overall, 165 survivors could not be reached by telephone or declined to participate, whereas 325 survivors (66.3\%) were interviewed face to face, most of them at home. There were no significant differences in gender or age between participants and non-participants. Most interviews $(95.4 \%)$ were conducted in November and December 2011. The interview was semistructured and was performed by health personnel. Prior to the interviews, training was provided regarding interviewing techniques in traumatised populations. The current needs of survivors for health services were assessed, and interviewers provided help in contacting adequate resources. The study was based on written consent and was approved by the Regional Committee for Medical and Health Research Ethics in Norway.

\section{Measures}

\section{Traumatic exposure}

A checklist was developed to assess 14 characteristics of potential traumatic exposure events ('yes' or 'no' answers) (Table 1). A sum score was constructed as a count of the number of 'yes' answers. Hearing gun shots was experienced by all survivors, and was hence excluded from the sum score, which ranged from 0 to 13 . In addition, we obtained information (registered as 'yes' or 'no' answers) about the following: physical injuries that needed medical care; admittance to hospital; current pain from physical injuries; and losing someone close during the attack, including the relationship to the deceased.

\section{Peritraumatic reactions}

Peritraumatic reactions, defined as emotional and physiological reactions experienced during or immediately after the event, were recorded using six items: fear, helplessness, horror, confusion, peritraumatic dissociation and rapid heartbeat, rated from 0 (not experienced at all) to 4 (experienced very much); a mean score was calculated (Cronbach's alpha 0.64).

\section{Social support}

Perceived social support was measured based on seven statements from the Duke-University of North Carolina Functional Social
Support Questionnaire (FSSQ), ${ }^{19}$ including the following: receiving attention, care and support from close friends and family, and being cared for by them if sick; receiving advice and support from others regarding school, work or personal matters; and being included in social activities with others. The statements had five response categories: 'As much as I would like' (5); 'Almost as much as I would like' (4); 'Somewhat, but would like more' (3); 'Less than I would like' (2); and 'Much less than I would like' (1). A mean score was calculated. The FSSQ has been shown to be valid and reliable, ${ }^{19,20}$ and exhibited acceptable internal consistency in the present study (Cronbach's alpha 0.80).

\section{Post-traumatic stress reactions}

Post-traumatic stress reactions over the past month were measured using the University of California at Los Angeles PTSD Reaction Index (PTSD-RI). ${ }^{21,22}$ The PTSD-RI is a 20 -item scale in which responses are recorded on a 5-point scale, ranging from 0 (never) to 4 (most of the time). Three items have two alternative formulations, and the highest score is applied to calculate the total score. Hence, 17 items make up the total symptom scale score, corresponding to DSM-IV-TR criteria for PTSD. ${ }^{23}$ Five items describe re-experiencing, seven items describe avoidance and five items describe increased arousal. The diagnostic criteria of PTSD were used to define levels of clinical PTSD. Reactions experienced 'much of the time' and 'most of the time' were defined as clinical symptoms. 'Full' PTSD diagnosis was based on meeting PTSD symptom criteria B, C and D. Meeting symptom criteria for only two subcategories was scored as 'partial' PTSD. ${ }^{22}$

In the present study, mean scores were computed and applied in the analyses. Cronbach's alpha was 0.89 for the total score. Because of the lack of a validated cut-off for the Norwegian population regarding the diagnostic level of PTSD, comparisons of post-traumatic stress reactions were made to a concurrent population-based study in Norway that included participants from 16 years of age. The general-population study included nine items from the PTSD-RI, ${ }^{24}$ including reactions of re-experiencing, avoidance and hyperarousal subsequent to the terror attacks. The selected items were suggested by the authors of the index (A. Steinberg, personal communication, 2013). The mean of the nine items correlated highly with the mean of the full scale in this sample $(r=0.95, P<0.001)$. For each of the survivors from the Utøya Island attack who were at least 16 years of age $(n=296)$, we calculated an expected score of post-traumatic stress reactions based on means from the general-population sample for each combination of age and gender. ${ }^{25}$ The general-population study included 599 individuals aged 16-56 years. The expected score could not be calculated for two individuals because of the lack of observations in the population study for that specific age and gender combination. This resulted in expected scores for 294 individuals.

\section{General mental health, functional impairment and life satisfaction}

The levels of depression and anxiety over the past 2 weeks were measured based on the eight-item version of the Hopkins Symptom Checklist-25 26,27 (SCL-8), rated on a scale from 1 (not bothered) to 4 (bothered a great deal), and applied as a mean score. Shorter versions of the SCL have shown good psychometric properties. ${ }^{28,29}$ In the present study, Cronbach's alpha was 0.85 for the total mean scale. Current functional impairment was measured based on three items that were designed for this study: having difficulty performing tasks, having less interest in activities than before and having difficulty in being with others. Items were rated on a 5-point scale, from 0 (not at all) to 4 (most of the 
time); Cronbach's alpha was 0.74. Life satisfaction was measured based on one item: 'How satisfied are you with life in general on a scale from 1 to 10,1 meaning very unsatisfied and 10 meaning very satisfied?'

\section{Sociodemographics}

We recorded whether participants had divorced parents, lived alone or were of non-Norwegian origin. Non-Norwegian origin was defined as having parents who were both born abroad. Parents' education was recorded in separate interviews with parents and was, therefore, available for a subsample of survivors $(n=174)$

\section{Statistics}

Pearson's $\chi^{2}$-tests were used for bivariate analyses of categorical variables, and Pearson's correlations were applied for continuous variables. Student's $t$-tests were used to compare mean differences between the two groups. No participants had more than two missing variables for the calculation of mean or sum scores, and mean scores were calculated based on the means of valid items within each scale. Associations between levels of post-traumatic stress reactions and independent variables were assessed using bivariate and multiple linear regression analyses. The sociodemographic parameters of having divorced parents and living alone were not included in the regression analyses, as these variables represent different challenges for different age groups in the sample. Parents' education was only available for a subsample $(n=174)$ and was also left out of the regression analyses. However, sensitivity analyses were performed to check whether adjustment for demographic variables altered the regression coefficients for the independent variables substantially. All tests were two tailed, with a significance level of $P \leqslant 0.05$. Statistical analyses were performed on a PC using IBM SPSS statistics, version 19. The $\mathrm{R}$ program was used to estimate the expected PTSD score based on the general-population study and to produce Fig. $1 .^{30}$

\section{Results}

The participants in this study comprised 153 females (47.1\%) and 172 males $(52.9 \%)$, who were all present on Utøya Island during the shooting on 22 July 2011 . The mean age was 19.4 years (s.d. $=4.6$ ) at the time of the shooting. The age range was $13-57$ years, although $92.5 \%$ of the participants were younger than 25 years of age and $97 \%$ were less than 30 years of age. The vast majority of the participants $(87.7 \%, n=285)$ were of Norwegian ethnic origin. Demographic variables by gender are shown in online Table DS1.

The survivors of the Utøya Island massacre were highly exposed to danger and experienced extreme trauma (auditory and visual) (Table 1).

We found no significant differences in trauma exposure between females and males. The mean number of reported event characteristics was 9.5 out of a possible 14 (s.d. $=2.2$; range $3-14$ ). In addition to the serious threat and having witnessed traumatic events on the island, $41 \%$ of the participants $(n=130)$ also felt threatened by the police, and 29.3\% $(n=95)$ were afraid of drowning.

A substantial minority was physically injured in the attack and needed medical care $(18.2 \%, n=59), 54.2 \%$ of whom $(n=32)$ were admitted to a hospital. The majority of survivors $(74.5 \%$, $n=240)$ lost someone close to them in the attack: $96.3 \%$ of them $(n=231)$ reported having lost a friend, $4.6 \%(n=11)$ reported having lost a boyfriend or girlfriend and $4.6 \%(n=11)$ reported having lost a family member. At follow-up, some survivors $(12.3 \%, n=40)$ reported current pain from the physical injuries that they had sustained during the attack.

Participants reported high levels of peritraumatic reactions, with a mean of 2.8 (s.d. $=0.8$ ) on a scale from 0 to 4 . Levels of peritraumatic reactions were significantly higher in females (mean 3.0 ; s.d. $=0.7$ ) compared with males (mean 2.7; s.d. $=0.8$; $P<0.001)$. The perceived social support reported was very high (mean 4.6; s.d. $=0.6$; on a scale of $0-5$ ). Social support did not differ significantly between the genders.

The mean score on the PTSD-RI scale (range 0-4) regarding current post-traumatic stress reactions in the survivors was 1.6 (s.d. $=0.7$ ), and was somewhat higher for the increased arousal subscale (mean 2.2; s.d. $=0.9$ ) than it was for re-experiencing (mean 1.5; s.d. $=0.9)$ and avoidance (mean 1.2; s.d. $=0.7$ ). In total, $47 \%$ of participants reported clinical levels of PTSD; $11 \%$ fulfilled the criteria for full PTSD diagnoses and 36\% for partial PTSD. The observed PTSD scores were compared with the expected scores that were calculated from the concurrent study of the general population, which included participants who were at least 16 years of age. ${ }^{24}$ For 294 of the Utøya Island survivors, an

Table 1 Characteristics of experiences of the event in the total sample according to gender $(n=325)$

\begin{tabular}{|c|c|c|c|c|c|}
\hline Event characteristics & Total, \% (n) & Females, \% (n) & Males, \% (n) & Odds ratio ${ }^{\mathrm{a}}(95 \% \mathrm{Cl})$ & $P$ \\
\hline Saw the terrorist or heard his voice & $73.1(237)$ & $69.3(106)$ & $76.6(131)$ & $0.69(0.42-1.13)$ & 0.138 \\
\hline Hid from or ran from the terrorist & 96.9 (314) & 96.7 (147) & $97.1(167)$ & $0.88(0.25-3.10)$ & 0.843 \\
\hline Heard gun shots & $100.0(325)$ & - & - & - & - \\
\hline Heard people screaming & $93.8(303)$ & $93.4(142)$ & $94.2(161)$ & $0.88(0.36-2.18)$ & 0.786 \\
\hline Smelled gunfire or other distinct smells & $34.4(109)$ & $35.1(53)$ & $33.7(56)$ & $1.06(0.67-1.69)$ & 0.798 \\
\hline Saw someone be injured or killed & $64.1(207)$ & $63.8(97)$ & $64.3(110)$ & $0.98(0.62-1.54)$ & 0.924 \\
\hline Heard someone be injured or killed & $82.6(265)$ & $84.8(128)$ & $80.6(137)$ & $1.34(0.75-2.41)$ & 0.326 \\
\hline Saw dead bodies & $86.7(280)$ & 84.3 (129) & $88.8(151)$ & $0.67(0.35-1.29)$ & 0.235 \\
\hline Touched dead bodies or injured people & 46.0 (149) & $46.7(71)$ & $45.3(78)$ & $1.06(0.68-1.64)$ & 0.806 \\
\hline Was afraid of being seriously injured & $77.5(251)$ & 79.7 (122) & 75.4 (129) & $1.28(0.76-2.17)$ & 0.356 \\
\hline Was afraid that he/she would die & 79.9 (259) & $84.3(129)$ & $76.0(130)$ & $1.67(0.97-2.97)$ & 0.064 \\
\hline $\begin{array}{l}\text { Saw the terrorist point the gun at him/her } \\
\text { or realised that he had shot at him/her }\end{array}$ & $45.1(146)$ & $44.7(68)$ & $45.3(78)$ & $0.98(0.63-1.51)$ & 0.912 \\
\hline Was afraid that he/she would drown & $29.3(95)$ & $29.4(45)$ & $29.2(50)$ & $1.01(0.63-1.63)$ & 0.973 \\
\hline Felt threatened by the police & $41.0(130)$ & $45.7(69)$ & $36.7(61)$ & $1.45(0.92-2.27)$ & 0.106 \\
\hline
\end{tabular}


expected score was calculated. The mean observed 9-item PTSD score for these 294 individuals was 1.8 (s.d. $=0.8$ ), compared with their expected 9-item PTSD score of 0.3 (s.d.=0.2); hence, their score was 6.3 times higher than that observed in the general population. Figure 1 displays histograms of observed and expected mean PTSD scores, as well as the ratio between the observed and expected scores. The distribution of expected scores based on the population study was highly skewed, as is normally observed for mental health symptoms in community samples. The scores observed in Utøya Island survivors were more normally distributed, and were similar to distributions found in clinical samples.

Figure 1 demonstrates the highly elevated symptom level in the survivors. A few survivors scored lower than expected; however, the vast majority scored several times higher.

Table 2 displays unadjusted and adjusted multiple associations between demographic variables, traumatic exposure, social support and post-traumatic stress reactions. Levels of post-traumatic stress reactions were significantly higher in females than in males, and in individuals with a minority ethnic background. This effect persisted in the adjusted model. The sum score corresponding to exposure levels during the shooting, levels of peritraumatic reactions, interpersonal loss, and current pain from injuries contributed independently to current post-traumatic stress reactions. Social support was highly and significantly associated with reduced post-traumatic stress reactions.

A major reduction in the regression coefficient for ethnicity was observed in the adjusted model. The regression coefficient for age remained low, although age was a significant predictor in the adjusted model. Sensitivity analyses showed that the regression coefficients were only marginally affected, with highly overlapping confidence intervals for all variables in the model when running the regression analyses only for participants under
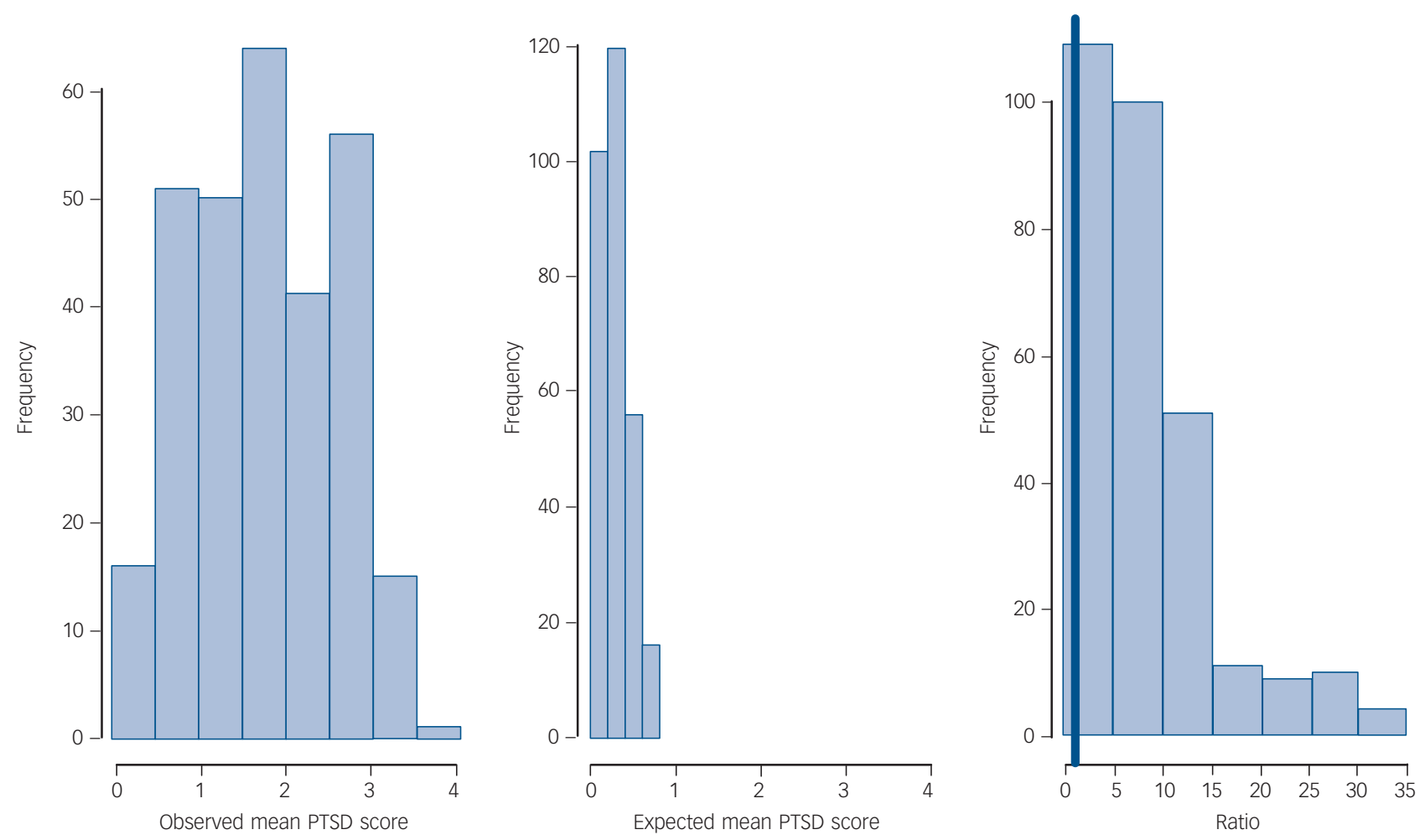

Fig. 1 Histograms of the observed post-traumatic stress disorder (PTSD) scores of survivors (left) compared with the expected levels based on PTSD scores from the general population (middle), and the ratio between the scores (right).

\begin{tabular}{|c|c|c|c|c|}
\hline & \multicolumn{2}{|l|}{ Bivariate } & \multicolumn{2}{|c|}{ Multivariate $(n=321)$} \\
\hline & Regression coefficient (95\% Cl) & $P$ & Regression coefficient (95\% Cl) & $P$ \\
\hline Gender ('female' indicated by 1) & $0.410(0.260$ to 0.560$)$ & $<0.001$ & 0.341 (0.215 to 0.466$)$ & $<0.001$ \\
\hline Age & $-0.015(-0.032$ to 0.001$)$ & 0.072 & $-0.015(-0.028$ to -0.002$)$ & 0.024 \\
\hline Ethnicity ('Norwegian' indicated by 0) & $0.600(0.372$ to 0.829$)$ & $<0.001$ & 0.291 (0.090 to 0.493 ) & 0.005 \\
\hline Sum of exposure (0-13) & $0.097(0.063$ to 0.131$)$ & $<0.001$ & 0.033 (0.003 to 0.063$)$ & 0.029 \\
\hline Peritraumatic reactions (0-4) & 0.384 (0.292 to 0.476$)$ & $<0.001$ & 0.230 (0.147 to 0.314$)$ & $<0.001$ \\
\hline Loss of someone close & 0.321 (0.143 to 0.498$)$ & $<0.001$ & 0.258 (0.117 to 0.399$)$ & $<0.001$ \\
\hline Current pain from injuries & 0.541 (0.310 to 0.772 ) & $<0.001$ & 0.308 (0.119 to 0.498$)$ & 0.002 \\
\hline Social support (1-5) & $-0.482(-0.608$ to -0.356$)$ & $<0.001$ & $-0.379(-0.492$ to -0.266$)$ & $<0.001$ \\
\hline
\end{tabular}




\begin{tabular}{|c|c|c|c|}
\hline & 1 & 2 & 3 \\
\hline \multicolumn{4}{|l|}{1 Post-traumatic stress reactions } \\
\hline 2 General mental health problems & $0.81^{* *}$ & & \\
\hline 3 Functional impairment & $0.67^{* *}$ & $0.65^{\star *}$ & \\
\hline 4 Life satisfaction & $-0.59 * *$ & $-0.63^{* *}$ & $-0.62^{* *}$ \\
\hline
\end{tabular}

the age of 25. Similarly, no substantial differences were identified when adjusting for parental education, parental divorce and living alone (not displayed in Table 2).

Post-traumatic stress reactions were highly and significantly associated with general mental health problems (mean 2.1; s.d. $=0.7$; range $1-4)$, functional impairment $\quad($ mean $=1.7$; s.d. $=0.9$; range $0-4$ ) and reduced life satisfaction (mean 6.8; s.d. $=2.0$; range $1-10)($ Table 3$)$.

\section{Discussion}

The survivors included in the present study all experienced horror and a threat to their lives. The brutality of the attack, the number of casualties and the horrifying experiences underscore the seriousness of the event. Such experiences may have a serious impact on the mental health of survivors ${ }^{10}$ and affect their families and close friends. ${ }^{31}$ However, comparison of the levels of post-traumatic stress reactions across events and populations is challenging; exposed populations differ with regard to, for example, prior traumatisation, standards of living, healthcare and social services. In addition, terrorist attacks differ in terms of the number of casualties, danger, the experience of witnessing a traumatic event, the duration of exposure to threat and trauma, level of destruction of infrastructure and access to necessary resources. $^{32,33}$ In this study, levels of post-traumatic stress reactions in survivors of the Utøya Island attack were compared with results from a concurrent study of the general Norwegian population. ${ }^{24}$ Almost all survivors reported elevated levels of post-traumatic reactions, with mean values that were six times higher than those observed in the general population. There is no reason to assume that the politically active adolescents and young adults present at the Utøya Island attack represented any particularly vulnerable group. Hence, we believe that the highly elevated symptoms observed are largely attributable to the traumatic experience and roughly reflect the mental health costs of the terrorist attack in the relatively early aftermath.

Even though all survivors were exposed to a life-threatening situation, the level of exposure to danger and having been a witness contributed uniquely to predicting the levels of posttraumatic stress reactions $4-5$ months after the disaster. Moreover, in line with an early exploration of the after-effects of school shootings ${ }^{7,34}$ and disaster studies of children and adolescents, ${ }^{33,35}$ peritraumatic emotional and physiological reactions predicted PTSD uniquely. Although males and females were equally exposed to a life-threatening situation, females reported higher levels of peritraumatic emotional and physiological reactions than did males. This may indicate that a heightened emotional and physiological response, and not necessarily appraisals of threat, contributes to elevated post-traumatic stress reactions in females. In addition to exposure characteristics, the loss of close friends was a significant predictor of post-traumatic stress reactions. The losses experienced in this study were highly traumatic, as many witnessed their best friends die and had strong sensory impressions of the death scenes. Such experiences can contribute to post-traumatic stress reactions in several ways; for example, by intrusive memories that are easily evoked by reminders, and potential shame and guilt, including survivor's guilt. ${ }^{15}$ Loss also represents a long-term strain because of grief reactions, longing for the dead person, meeting with bereaved families and friends in the community and possibly having to rearrange one's social life.

Interestingly, current pain (4-5 months after the attack) resulting from injuries from the attack contributed uniquely to post-traumatic stress reactions. Similar results were obtained in a study of trauma patients in an intensive care unit. ${ }^{36}$ Pain may often lead to impaired functioning, serve as a reminder of the traumatic event and trigger reactions such as sadness, hopelessness, anger and intrusive memories.

Our study showed that almost all survivors perceived high levels of social support, and that social support was highly and significantly associated with reduced post-traumatic stress reactions. This finding is encouraging and indicates that care and support from close family and friends makes an important difference, even after exceptionally horrifying events, and that outreach after traumatic events should not be considered a purely professional task.

Youths of non-Norwegian origin seemed to constitute a vulnerable group for developing post-traumatic stress reactions, even after controlling for the effect of social support. Similar results were obtained in the study of the general Norwegian population. ${ }^{24}$ Studies of highly exposed groups after the $9 / 11$ attacks in the USA have also identified ethnic background as a factor that was associated with subsequent PTSD symptoms. ${ }^{2,34}$ The mechanisms underlying these associations are not clear; however, they may be related to a perception of ongoing threat against ethnic minority groups. There are also several other plausible explanations for these findings, such as socioeconomic differences, previous traumatic experiences or differences in social integration. Further studies of terrorist attack victims are needed to determine why people from ethnic minority groups seem to be more vulnerable to mental health problems.

Although post-traumatic stress reactions are considered to be the most typical reactions to traumatic events, other psychological reactions are common. In our study, post-traumatic stress reactions, anxiety and depression symptoms, reduced levels of functioning and reduced life satisfaction were significantly associated (Table 3), indicating that the psychological state of these youths may have had a considerable impact on their ability to work, study and maintain a normal social life.

\section{Strengths and limitations}

One of the strengths of this study was the relatively high response rate and very low levels of missing data compared with other studies of traumatic events. In the acute aftermath of a disaster, 
survivors may be reluctant to participate in research because of the adversities and challenges they face post-trauma. In this study, change of school and residence impeded recruitment. However, non-responders did not differ significantly from responders in age and gender. The level of exposure was relatively uniform across the participants, which enabled us to study the relative importance of other factors for long-term mental health. Furthermore, the study was performed in an early phase after the disaster, and our findings add to the limited knowledge of victims' health in the acute aftermath. The fact that the PTSD-RI was administered by trained professionals as part of the face-to-face interview was another strength of the study, as it ensured that the scores reflected valid post-traumatic stress reactions. The PTSD-RI is one of the instruments used most widely for assessing post-traumatic stress reactions in adolescents, and has been applied in other Norwegian studies. ${ }^{33}$

Despite these strengths, our findings must be considered in light of several limitations. Due to the cross-sectional design of the study, causal relationships could not be established. The experiences of this specific population have limited generalisability to other affected populations, but are useful for situations involving shootings and youths. Lack of measures of pre-trauma health conditions, demographic information and psychosocial interventions post-trauma may have influenced the outcome of the study. A comprehensive diagnostic instrument for assessing depression and anxiety would have added solidity to the assessments of psychological health; however, because of practical constraints, such as the limited time for training interviewers and ethical considerations concerning the length of the interview, this was not considered feasible. The general-population scores for post-traumatic stress reactions were based on a more limited measurement of PTSD (nine items). However, the correlation between the mean PTSD scores on the 17-item and 9-item versions of the PTSD-RI was very high. The general-population study was timed to match the data collection in the present study, and the nine PTSD items were identical and were asked in an identical manner. However, the response rate differed between the two studies: it was much lower in the population study (the details of that study, response rates and extensive analyses of the non-responders are published elsewhere) ${ }^{24}$ In addition, the data collection in the population study was performed by telephone interviews, whereas face-to-face interviews were performed in the Utøya Island study. Hence, some limitations to the comparisons between observed and expected scores should be considered. At the same time, we do not expect that a higher response rate or a face-to-face interview mode would change the results substantially.

It is also worth mentioning that, in this study, interviewers were health professionals who were trained for the situation, and supervision was provided throughout the data collection. Survivors in need of professional help were given advice and help in contacting health and social services.

\section{Clinical implications}

The sixfold increase in mental health problems in survivors compared with the general population illustrates the high mental health cost of this brutal attack. The level of suffering in the early aftermath called for a national outreach programme including first-line services and targeted interventions for those in need. Development of mental health symptoms was largely attributable to the traumatic experience, and other high-impact traumas such as school shootings, torture, hostage situations and other violent crimes may affect groups of survivors similarly. For high-impact traumas, outreach programmes should include easy access first-line services and interventions and adequate assessments over time to target those in need of further treatment. In addition, social support from friends, family and others may protect against further suffering, and should be emphasised in all information materials provided after disasters.

\section{Grete Dyb, MD, PhD, Norwegian Centre for Violence and Traumatic Stress Studies, and Institute of Clinical Medicine, Faculty of Medicine, University of Oslo; Tine K. Jensen, PhD, Egil Nygaard, PhD, Norwegian Centre for Violence and Traumatic Stress Studies, and Department of Psychology, Faculty of Social Sciences, University of Oslo; Øivind Ekeberg, MD, PhD, Department of Acute Medicine, Oslo University Hospital, and Department of Behavioural Sciences in Medicine, Institute of Basic Medical Sciences, Faculty of Medicine, University of Oslo; Trond H. Diseth, MD, PhD, Institute of Clinical Medicine, Faculty of Medicine, University of Oslo, and Department of Clinical Neurosciences for Children, Women and Children's Division, Oslo University Hospital; Tore Wentzel-Larsen, MSc, Norwegian Centre for Violence and Traumatic Stress Studies, and Center for Child and Adolescent Mental Health, Eastern and Southern Norway; Siri Thoresen, PhD, Norwegian Centre for Violence and Traumatic Stress Studies, Oslo, Norway}

Correspondence: Grete Dyb, MD, PhD, Norwegian Centre for Violence and Traumatic Stress Studies, Postbox 181 Nydalen, 0409 OSLO, Norway. Email: grete.dyb@nkvts.unirand.no

First received 31 May 2013, final revision 15 Aug 2013, accepted 5 Sep 2013

\section{Funding}

This study was funded by the Norwegian Directorate of Health.

\section{References}

1 Bongar B. The psychology of terrorism: defining the need and describing the goals. In Psychology of Terrorism (eds B Bongar, LM Brown, LE Beutler, JN Breckenridge, PG Zimbardo): 3-12. Oxford University Press, 2006.

2 Neria Y, DiGrande L, Adams BG. Posttraumatic stress disorder following the September 11, 2001, terrorist attacks: a review of the literature among highly exposed populations. Am Psychol 2011; 66: 429-46.

3 Galea S, Vlahov D, Tracy M, Hoover DR, Resnick H, Kilpatrick D. Hispanic ethnicity and post-traumatic stress disorder after a disaster: evidence from a general population survey after September 11, 2001. Ann Epidemiol 2004; 14: $520-31$.

4 Neria $Y$, Wickramaratne $\mathrm{P}$, Olfson $\mathrm{M}$, Gameroff MJ, Pilowsky DJ, Lantigua R, et al. Mental and physical health consequences of the September 11, 2001 (9/11) attacks in primary care: a longitudinal study. J Trauma Stress 2013; 26: 45-55.

5 Hansen MB, Nissen A, Heir T. Proximity to terror and post-traumatic stress: a follow-up survey of governmental employees after the 2001 Oslo bombing attack. BMJ Open 2013; 3: e002692.

6 Scrimin S, Axia G, Capello F, Moscardino U, Steinberg AM, Pynoos RS. Posttraumatic reactions among injured children and their caregivers 3 months after the terrorist attack in Beslan. Psychiatry Res 2006; 141: 333-6.

7 Pynoos RS, Frederick C, Nader K, Arroyo W, Steinberg A, Eth S, et al. Life threat and posttraumatic stress in school-age children. Arch Gen Psychiatry 1987; 44: 1057-63.

8 Schwarz ED, Kowalski JM. Malignant memories: PTSD in children and adults after a school shooting. J Am Acad Child Adolesc Psychiatry 1991; 30: 936-44.

9 Trappler B, Friedman S. Posttraumatic stress disorder in survivors of the Brooklyn Bridge shooting. Am J Psychiatry 1996; 153: 705-7.

10 Galea S, Nandi A, Vlahov D. The epidemiology of post-traumatic stress disorder after disasters. Epidemiol Rev 2005; 27: 78-91.

11 Suomalainen L, Haravuori $\mathrm{H}$, Berg N, Kiviruusu O, Marttunen M. A controlled follow-up study of adolescents exposed to a school shooting - psychological consequences after four months. Eur Psychiatry 2011; 26: 490-7.

12 Alisic E, Jongmans MJ, van Wesel F, Kleber RJ. Building child trauma theory from longitudinal studies: a meta-analysis. Clin Psychol Rev 2011; 31: $736-47$

13 Salmon K, Bryant RA. Posttraumatic stress disorder in children. The influence of developmental factors. Clin Psychol Rev 2002; 22: 163-88.

14 Trickey D, Siddaway AP, Meiser-Stedman R, Serpell L, Field, AP. A metaanalysis of risk factors for post-traumatic stress disorder in children and adolescents. Clin Psychol Rev 2012; 32: 122-38. 
15 Brewin CR, Andrews B, Valentine JD. Meta-analysis of risk factors for posttraumatic stress disorder in trauma-exposed adults. $J$ Consult Clin Psychol 2000; 68: 748-66.

16 Charuvastra A, Cloitre M. Social bonds and posttraumatic stress disorder. Annu Rev Psychol 2008; 59: 301-28.

17 Ozer, EJ, Best SR, Lipsey TL, Weiss DS. Predictors of posttraumatic stress disorder and symptoms in adults: a meta-analysis. Psychol Bull 2003; 129 52-73.

18 Comer JS, Kendall PC. Terrorism: the psychological impact on youth. Clin Psychol Sci Prac 2007; 14: 179-212.

19 Broadhead WE, Gehlbach SH, de Gruy FV, Kaplan BH. The Duke-UNC functional social support questionnaire. Measurement of social support in family medicine patients. Med Care 1988; 26: 709-23.

20 Epinoa HM, Rich ML, Kaigamba F, Hakizamungu M, Socci AR, Bagiruwigize E, et al. Reliability and construct validity of three health-related self-report scales in HIV-positive adults in rural Rwanda. AIDS Care 2012; 24: 1576-83.

21 Pynoos RS, Rodriguez N, Steinberg AM, Stuber M, Frederick C. UCLA PTSD Index for DSM-IV. University of California, 1998.

22 Steinberg AM, Brymer MJ, Decker KB, Pynoos RS. The University of California at Los Angeles post-traumatic stress disorder reaction index. Curr Psychiatry Rep 2004; 6: 96-100.

23 American Psychiatric Association. Diagnostic and Statistical Manual of Mental Disorders (4th edn, Text Tevision) (DSM-IV-TR). APA, 2000

24 Thoresen S, Aakvaaq HF, Wentzel-Larsen T, Dyb G, Hjemdal OK. The day Norway cried: proximity and distress in Norwegian citizens following the 22nd July 2011 terrorist attacks in Oslo and on Utøya Island. Eur J Psychotraumatol 2012; 3: 19709.

25 Hjermstad MJ, Fayers PM, Bjordal K, Kaasa S. Using reference data on quality of life - the importance of adjusting for age and gender, exemplified by the EORTC QLQ-C30 (+3). Eur J Cancer 1998; 34: 1381-9.
26 Solberg Ø, Dale MT, Holmstrøm H, Eskedal LT, Landolt MA, Vollrath ME. Long-term symptoms of depression and anxiety in mothers of infants with congenital heart defects. J Pediatr Psychol 2011; 36: 179-87.

27 Derogatis LR, Lipman RS, Rickels K, Uhlenhuth EH, Covi L. The Hopkins Symptom Checklist (HSCL): a self-report symptom inventory. Behav Sci 1974; 19: $1-15$

28 Strand BH, Dalgard OS, Tambs K, Rognerud M. Measuring the mental health status of the Norwegian population: a comparison of the instruments SCL-25, SCL-10, SCL-5 and MHI-5 (SF-36). Nord J Psychiatry 2003; 57: 113-8.

29 Tambs $K$, Moum T. How well can a few questionnaire items indicate anxiety and depression? Acta Psychiatr Scand 1993; 87: 364-7.

30 Team RC. R: A Language and Environment for Statistical Computing. R Foundation for Statistical Computing, 2013.

31 Norris FH. Impact of mass shootings on survivors, families and communities. PTSD Res Quart 2007; 18: 1-8.

32 North CS. A tale of two studies of two disasters: comparing psychosocial responses to disaster among Oklahoma City bombing survivors and Hurricane Katrina evacuees. Rehabil Psychol 2010; 55: 241-6.

33 Jensen TK, Dyb G, Nygaard E. A longitudinal study of posttraumatic stress reactions in Norwegian children and adolescents exposed to the 2004 tsunami. Arch Pediatr Adolesc Med 2009; 163: 856-61.

34 Schwartz ED, Kowalski JM. Malignant memories: PTSD in children and adults after a school shooting. J Am Acad Child Adolesc Psychiatry 1991; 30 936-44.

35 Dyb G, Jensen TK, Nygaard E. Children's and parents' posttraumatic stress reactions after the 2004 tsunami. Clin Child Psychol Psychiatry 2011; 16: 621-34.

36 Tøien K, Myhren H, Bredal IS, Skogstad L, Sandvik L, Ekeberg Ø. Psychological distress after severe trauma: a prospective 1-year follow-up study of a trauma intensive care unit population. J Trauma 2010; 69: 1552-9.

\title{
poems by doctors
}

\section{Not Transference}

\author{
Jacob Freedman
}

I wouldn't do my teachers right

If I didn't address it with you in today's session

And ask what was going on inside your mind

When you nearly ran me over earlier this morning

I know that it was still before sunrise

And that I was riding in the middle of the road

And I'II admit that I forgot to wear my reflective vest

But I would have figured you'd recognise your psychiatrist

Perhaps it isn't a transference reaction

And it's not because I brought up the late payments

Or because I've been pushing you to talk with your mother

Thank you for reminding me to use my bicycle's safety light 